\title{
Combining Semantic Tools for Automatic Evaluation of Alternative Texts
}

\author{
Carlos Duarte \\ Carlos M. Duarte \\ LASIGE, Faculdade de Ciências, Universidade de Lisboa \\ Campo Grande, Lisboa, Portugal \\ caduarte@fc.ul.pt \\ cmduarte@lasige.di.fc.ul.pt \\ Luís Carriço \\ lmcarrico@fc.ul.pt
}

\begin{abstract}
Automated accessibility evaluation will have an increasingly important role in the near future due to legal requirements to monitor the accessibility of the websites of public bodies in the European Union. However, automated evaluation tools are still limited in the scope of the conformance testing they are able to perform. Future solutions resorting to AI based techniques might help in addressing some of these limitations. In this paper we present an updated version of an algorithm that classifies the quality, from an accessibility perspective, of alternative texts for images in web pages. We evaluated the proposed algorithm on a set of 149 images and their corresponding alternative texts and found improved performance compared to the initial version of the algorithm.
\end{abstract}

\section{CCS Concepts}

- Human-centered computing Accessibility design and evaluation methods

\section{Keywords}

Automatic accessibility evaluation; semantic analysis; images; alternative text; algorithm.

\section{INTRODUCTION}

Web accessibility evaluation is an important process for web content developers to ensure the content they create is accessible for most, if not all, of the potential consumers or users of that content. Furthermore, accessibility evaluation is also now relevant for monitoring bodies. For example, in Europe following the publication of the European Directive on the accessibility of websites and mobile applications of public sector bodies [4], national monitoring agencies will have to ensure that websites of public bodies abide by the national laws that transpose the directive. Given the number of websites that need to be monitored, it can be expected that automated means for monitoring the accessibility of the websites will be needed.

Existing automated accessibility evaluation tools provide several benefits, including reduced costs, quicker availability of results, higher consistency, and others [8]. However, those tools also possess several limitations. One of the most relevant is their inability to assess all the guidelines that define what is an

Permission to make digital or hard copies of part or all of this work for personal or classroom use is granted without fee provided that copies are not made or distributed for profit or commercial advantage and that copies bear this notice and the full citation on the first page. Copyrights for third-party components of this work must be honored. For all other uses, contact the Owner/Author.

W4A '19, May 13-15, 2019, San Francisco, CA, USA.

(C) 2019 Copyright is held by the owner/author(s).

ACM ISBN 978-1-4503-6716-5/19/05.

https://doi.org/10.1145/3315002.3317558 accessible website, according to the directive [9].

Ways to improve both the coverage as well as the accuracy of evaluations have been studied in the past. Recently, AI based solutions have been gaining some traction [1]. One approach proposed by Duarte et al. [3] consisted in using existing tools that perform image analysis and compute semantic similarity to address the problem of assessing the quality of text alternatives for image-based content. This is a specific example of the difficulties that automated tools face. Most existing solutions are able to detect the presence of the alternative text but cannot judge its quality. Instead, they redirect the assessment to a human expert. In this paper, we present an updated version of the algorithm tackling this problem. In the next section we discuss the attributes that an alternative text should possess. This is followed by the description of the updated algorithm. After we present an evaluation of the algorithm, and in the final section we conclude and discuss the current limitations of our approach.

\section{IMAGE DESCRIPTIONS}

Images have always been a paramount factor in the web. The web's popularity really started growing in 1993 due to the inclusion of the IMG tag in the NCSA Mosaic browser, which allowed mixing text and images on the same page, capturing the interest of the tech press [11]. Nowadays, with the growth of social networks and user generated content, image uploads are in the order of billions per day [6]. If users and content providers don't make their images accessible, this represents an enormous amount of information that is inaccessible to a significant part of the population of web users. Julie Grundy's report [5] sheds some light on the types of problems that alternative texts for images can present: $66.36 \%$ of the problems found were informative images that did not have a text alternative; $20.33 \%$ corresponded to informative images with a text alternative that was inadequate or not useful; $10.35 \%$ were decorative images with an alternative text when it was not required; and $2.96 \%$ matched images with a text alternative that repeated other available content.

This report highlights that not all images should be treated equally. The W3C groups images according to their purpose [12]. Informative images have the purpose to convey additional information or concepts to the user. The text alternative for these images should convey the same meaning that is displayed visually. Typically, a literal description of the image is not required to convey its meaning. Decorative images do not add new information to a page. These images might repeat content that is available in text adjacent to the image or might be used simply to make the website more appealing from a visual perspective. A null alternative text should be provided for these images so that they can be ignored by assistive technology. Functional images initiate actions instead of providing information. They are commonly used in interactive elements, like 
buttons. The text alternative should convey the action, not a description of the image.

The instructions above provide a brief guidance on writing text alternatives. Other resources provide more details on what a good text alternative for image content should be [7, 10]. The following list summarizes the key attributes for a text alternative for informative images:

- The description should be succinct, with some sources placing the maximum size around 125 characters;

- The alternative text should convey the most relevant concepts from the image;

- The larger the number of relevant image concepts conveyed in the text alternative, the more likely it is to be a good description of the image;

- The alternative text must take into account the context of the webpage the image is in;

- The alternative text must not provide redundant information that is already available in the webpage;

- Avoid using text alternatives beginning with "images of" or "graphic of".

\section{SEMANTIC ANALYSIS}

In [3] we presented an algorithm with the same goal. That algorithm related concepts extracted from an image with the text of its description, identified topic domains which were related to both the image's concepts and the description, and identified named entities in the description that were related to those domains. The algorithm combined all these inputs into a semantic measure of the quality of the image's description. The algorithm was evaluated on a set of 15 images and corresponding descriptions. The algorithm's classification was compared with the classification of 101 survey participants and a moderate correlation was found between both classifications $(\rho=0.404)$.

In the updated algorithm presented here, we kept the assessment of direct and domain relations, added relations based on image's metadata when available and included a penalty factor based on the description's size.

\subsection{The SCREW v2 Algorithm}

The algorithm takes as input an image and its description and has as output a classification between 0 and 1 representing the quality of the image's description from an accessibility perspective. The algorithm combines three levels of concept comparison.

The first level compares the image descriptors with the image's description. The image descriptors are the output of an image analysis module. For each image descriptor $I d_{k}$ in the set of image descriptors $I d$, the algorithm searches the descriptor (and its plural form) in the description. If the descriptor is found, the relations counter $C R$ is incremented. If the descriptor is not found in the description, the algorithm searches the description for a synonym $S I d_{k}$ of the descriptor. If the synonym is found, the relations counter $C R$ is incremented. If the synonym is not found, the algorithm assesses if the semantic relation between the descriptor and the description is above a threshold $t S R_{l}$. If the semantic relation is above the threshold, the relations counter $C R$ is incremented. The classification output from this level is equal to $C R$ divided by the number of descriptors in the set $I d$.

The second level compares the image's domain descriptors with the image's description. The image analysis module can output general descriptors (used in the first level comparison) or it can output descriptors related to specific domains (e.g. food). The algorithm begins by assessing the semantic relation between each image descriptor $I d_{k}$ and each domain name $D_{j}$. If the value of the semantic relation is above a threshold $t S R_{2}$, the image analysis module is run on the specific domain, resulting in a set $D d$ of image domain descriptors $D d_{k}$. For each domain descriptor $D d_{k}$ not already present in the set $I d$, the algorithm begins by searching for it in the description. If it is found, a direct relation increment $i D R$ is added to the classification. It is not found, the algorithm searches the description for a synonym of the domain descriptor (or of its plural form). If the synonym $S D d_{k}$ is found in the description, the classification is incremented by the synonym relation increment $i S R$. If no synonym is found, the algorithm checks if the semantic relation between the domain descriptor and the description is above a threshold $t S R_{3}$. If it is, a threshold increment $i T H$ is added to the classification.

The third level compares image metadata with the image's description. Images, especially photos, can have metadata, either automatically captured by the camera or manually added by the photographer. This metadata comprises different attributes. Whatever the source, the metadata represents information about the image that might not be available from other sources. Our algorithm considers the following attributes when they are present in the image: keywords, country, city and sub-location. The algorithm searches each of these attributes $M d_{k}$ in the description. If the attribute is found, the classification is increased by an increment $i D M$. If it is not found, the algorithm extracts named entities (e.g. the name of persons or of places) from the description and assesses the semantic relation between the attributes and the named entities. If any semantic relation is found above a threshold $t S R_{4}$, an increment $i N E$ is added to the classification.

The final step of the algorithm judges the size of the description. Based on the quality of the descriptions of images in the training set (described in the following section) we created a bandpass filter that penalizes descriptions as a function of their size (Figure 1). The classification resulting from the output of the first level with the addition of all increments from the second and third levels is multiplied by the description size quality factor. If the final output is above 1, the algorithm limits the output to 1 .

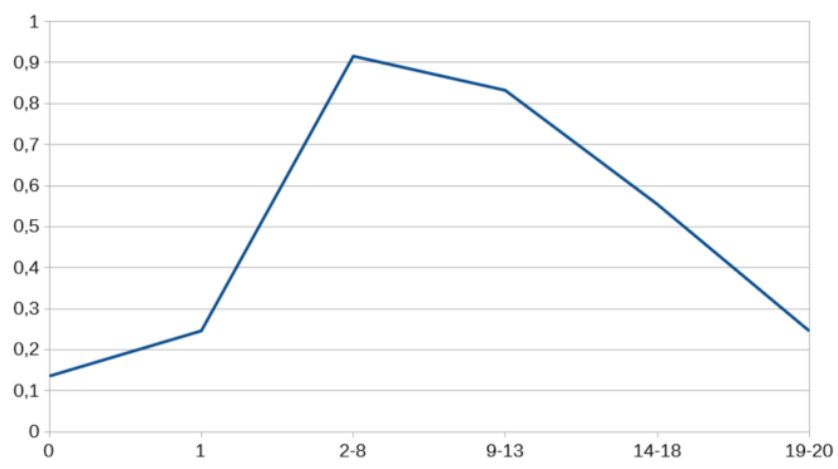

Figure 1 - Description quality (vertical axis) as function of the number of words in the image's description (horizonal axis)

The following pseudo-code presents the described algorithm.

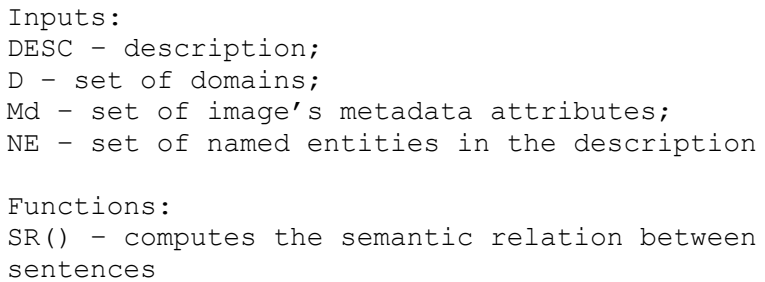




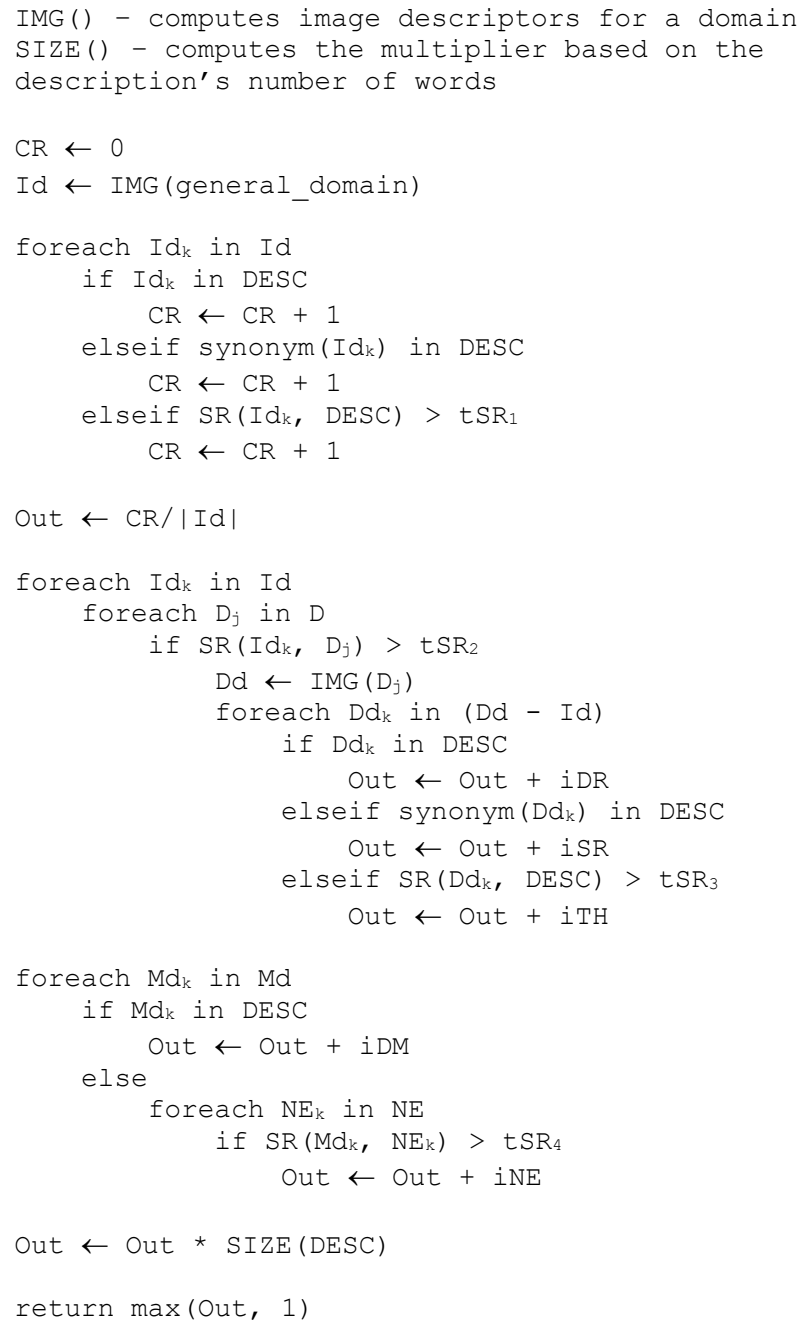

\subsection{Parameter Optimization}

To optimize the different parameters and thresholds used in the algorithm, we created a training set. A set of 45 images and corresponding text alternative were collected from several news sites and blogs. A listing of the image's sources, the URL of the images and their alt-text can be found at https://goo.gl/8nX25s One of the authors classified the relation between each image and its alt-text according to the heuristics presented in Section 2 . The classification is presented also in the above link in two scales, from 0 to 1 and from 1 to 4 . Fourteen of the relations were classified as very bad, 15 as bad, 8 as good and 8 as very good. The average of the relation classifications was 2.15 .

Table 1. Optimized algorithm parameters

\begin{tabular}{|c|c|c|c|}
\hline Parameter & Value & Parameter & Value \\
\hline$t S R_{1}$ & 0.21 & $t S R_{2}$ & 0.5 \\
\hline$t S R_{3}$ & 0.21 & $t S R_{4}$ & 0.5 \\
\hline$i D R$ & 0.35 & $i S R$ & 0.15 \\
\hline$i T H$ & 0.10 & $i D M$ & 0.35 \\
\hline$i N E$ & 0.15 & & \\
\hline
\end{tabular}

We run the algorithm with multiple combinations of values for the parameters (each parameter was tested with values from 0 to 1 with increments of 0.05). The configuration with the highest correlation ( $\rho=0.683$ ) between the algorithm's classification and the author's classification is presented in Table 1.

For the situations where, instead of a continuous value between 0 and 1, a binary classification (good vs. bad description) is needed, we optimized the threshold value for a good classification by finding the value that maximized the number of correct classifications. Classifier values equal or above 0.3 represent good descriptions, while values below represent bad descriptions. With this optimization, the algorithm correctly classifies 36 of the 45 images (an 80\% accuracy) in the training set.

\subsection{Semantic Services}

The algorithm described relies on a set of semantic functions. In our implementation of the algorithm we used free applications and services for the required functions. These applications are described in the following.

To identify descriptors of images we used the Clarifai ${ }^{1}$ service. Clarifai uses different recognition models to extract information from images. The general model is used by us to extract the general descriptors. The domain models are used to extract the domain descriptors. We used the following Clarifai domain models: Apparel, Celebrities, Food, Travel, and Wedding.

To identify semantic relations between descriptors and descriptions we used the Swoogle ${ }^{2}$ service. This service, developed at UMBC, is able to compute the semantic similarity between two sentences. Swoogle can use different corpus for similarity calculation. In our test we identified the webbase corpus as the one with better results for our aims.

To identify named entities in the descriptions we used the $\mathrm{spaCy}^{3}$ library. spaCy is a natural language processing library, with statistical models supporting eight different languages. It offers more features, but we only used named entity recognition.

To compute the semantic similarity between named entities and image attributes we used the Sematch ${ }^{4}$ framework. Sematch's service is similar to what Swoogle offers, but we found its performance was better when named entities were involved in the semantic similarity computation.

\section{EVALUATION}

To evaluate the performance of the proposed algorithm we created a new image set. This test set included 149 images collected from blogs, online magazines, news sites and other sources. None of the images of the training set was included in the test set. The test set is available at https://goo.gl/7CZMko with information of the page where the image was found and the URL of the image's alttext. All the images were classified individually by each of the authors in a scale from 1 to 4 , with 1 representing a very bad description, 2 a bad description, 3 a good description and 4 a very good description. As the agreement between classifications was not satisfactory, the authors arranged a meeting where they reached a consensus classification. Individual and consensus classifications can also be found in the link provided.

The correlation between the expert consensus classification and the algorithm classification was 0.698 , a strong correlation.

\footnotetext{
${ }^{1}$ https://clarifai.com

${ }^{2}$ http://swoogle.umbc.edu/SimService/api.html

${ }^{3}$ https://spacy.io

${ }^{4}$ http://gsi-upm.github.io/sematch/
} 
We also analyzed the performance with a binary classification (good vs bad). We used the 0.3 threshold for the classifier to separate good and bad classifications. Table 2 presents the number of true positives, true negatives, false positives and false negatives.

Table 2. Classifier performance

\begin{tabular}{|c|c|c|c|}
\hline True Positives (TP) & 58 & True Negatives (TN) & 66 \\
\hline False Positives (FP) & 10 & False Negatives (FN) & 15 \\
\hline
\end{tabular}

From the total of 149 classifications, $83 \%$ are true positives or negatives. Table 3 presents several classifier metrics.

Table 3. Classifier metrics

\begin{tabular}{|c|c|c|c|}
\hline Accuracy & 0.832 & Precision & 0.853 \\
\hline Sensitivity & 0.795 & Specificity & 0.815 \\
\hline F-measure & 0.823 & & \\
\hline
\end{tabular}

All metrics present similar values. In particular, we can see from the sensitivity and specificity values that the algorithm's performance in correctly identifying actual positives and actual negatives is similar and can be considered positive.

Finally, we computed the level of agreement between the algorithm and the experts' classifications using Cohen's Kappa. The value for Kappa is 0.664 representing good agreement between the classifications [2].

\section{CONCLUSIONS}

In this paper we presented the second version of SCREW, an algorithm to automatically assess the quality of text alternatives for images from a web accessibility perspective. The algorithm explores semantic relations between different descriptors of the image content and the available text alternative. The descriptors considered include the ones extracted by image analysis models, possibly limited to specific domains of analysis, and image's metadata that might be captured automatically by the camera (if it is a photo) or entered manually by the image's creator. The algorithm was evaluated on a set of 149 images. The correlation between the algorithm's classification in a 1 to 4 scale and a classification originating from the consensus of three experts was 0.698. When considering just a binary scale, the algorithm's accuracy was 0.83 , revealing a positive performance for classifying both good and bad descriptions.

Even though these developments show promise in the use of automatic tools for performing what currently is a manual evaluation process, there are still limitations that need to be addressed: first, the quality of the results depends directly of the quality of the semantic services used. Currently the results are already positive but can still be improved if the semantic services capabilities are also improved. A second limitation, linked to the first one, relates to the domains currently being used. These domains are limited to those provided by the image analysis service used, and do not cover all possible domains. The lack of availability of these services in more languages than English is a further limitation. Another level of limitations stems from the lack of usage of the page's context. Currently, we are analyzing the image and its description without considering the remainder of the page's content. This precludes us from penalizing descriptions that are redundant when considering adjacent content, for example. Furthermore, the algorithm currently only applies to informative images, but does not include mechanisms to check if the purpose of the image is informative or another. Nevertheless, even with all the current limitations, the algorithm can be useful in a semi-automatic web accessibility evaluation scenario, representing an improvement over current solutions that mark all alt-texts as something that needs to be checked by a human. The classification provided by the algorithm can represent a confidence level that can be used by both developers to find the descriptions that are, probably, in higher need of reformulation, and human accessibility evaluators to find a subset of images of a site to manually check their conformance to guidelines.

\section{ACKNOWLEDGMENTS}

This paper was written with support from the WAI-Tools Project, co-funded by the European Commission (EC) H2020 GA 780057. This work was supported by FCT through funding of the LASIGE Research Unit, ref. UID/CEC/00408/2019.

\section{REFERENCES}

[1] Abou-Zahra, S., Brewer, J., and Cooper, M. 2018. Artificial Intelligence for Web Accessibility-Conformance Evaluation as a Way Forward? In W4A'18 (Lyon, France). ACM. DOI= https://doi.org/10.1145/3192714.3192834

[2] Altman, D.G. 1990. Practical statistics for medical research. CRC press.

[3] Duarte, C., Matos, I., and Carriço, L. 2018. Semantic Content Analysis Supporting Web Accessibility Evaluation. In W4A'18 (Lyon, France). ACM. DOI= https://doi.org/10.1145/3192714.3196828

[4] European Union. 2016. Directive (EU) 2016/2102 of the European Parliament and of the Council of 26 October 2016 on the accessibility of the websites and mobile applications of public sector bodies. http://data.europa.eu/eli/dir/2016/2102/oj

[5] Grundy, J. 2016. Three common accessibility pitfalls for developers: text alternatives. https://simplyaccessible.com/article/three-pitfalls-textalternatives/

[6] Marr, B. 2018. How Much Data Do We Create Every Day? The Mind-Blowing Stats Everyone Should Read. https://www.forbes.com/sites/bernardmarr/2018/05/21/howmuch-data-do-we-create-every-day-the-mind-blowing-statseveryone-should-read/\#61babda560ba

[7] Moz. 2018. What is Alt Text? Alt Tags for Images. https://moz.com/learn/seo/alt-text

[8] Parmanto, B. and Zeng, X. 2005. Metric for web accessibility evaluation. J. Assoc. Inf. Sci. Tech. 56, 13 (Aug. 2005), 1394-1404. DOI= http://dx.doi.org/10.1002/asi.20233

[9] Vigo, M., Brown, J., and Conway, V. 2013. Benchmarking web accessibility evaluation tools: measuring the harm of sole reliance on automated tests. In W4A'13, Rio de Janeiro, Brazil, 2013. DOI= https://doi.org/10.1145/2461121.2461124

[10] WebAIM. 2018. Alternative Text. https://webaim.org/techniques/alttext/

[11] Weber, M. 2018. Browsers and Browser Wars. The SAGE Handbook of Web History. SAGE Publications.

[12] W3C. 2017. Images Concepts. https://www.w3.org/WAI/tutorials/images/ 